$$
f(x)=\int_{0}^{\infty} x(t) d p(t)
$$

$x \varepsilon S$.

Now (4.1) is a linear functional on $R$, and consequently a linear functional on $S$. Hence (4.2) states that every distributive functional on $S$ is linear; but this is impossible unless $S$ is finite-dimensional,* which it is not. This contradiction establishes the theorem.

Norton, Massachusetts

\title{
ON FUNDAMENTAL SYSTEMS OF SYMMETRIC FUNCTIONS $\uparrow$
}

\section{H. T. ENGSTROM}

A set $S$ of $n$ polynomials over a field $K$, symmetric in $n$ variables, $x_{1}, x_{2}, \cdots, x_{n}$, is said to form a fundamental system if any rational function over $K$, symmetric in these variables, can be expressed rationally in terms of the polynomials of $S$. In this paper we show that any $n$ algebraically independent symmetric polynomials over a field $K$ of characteristic zero form a fundamental system if the product of their degrees is less than $2 n$ !.

The result follows from a theorem due to Perron : $\ddagger$

Theorem 1. Between $n+1$ polynomials (not constant), $f_{1}, f_{2}, \cdots, f_{n+1}$, in $n$ variables, of degrees $m_{1}, m_{2}, \cdots, m_{n+1}$, respectively, there is always an identity of the form

$$
\sum C_{\nu_{1} \nu_{2}} \cdots \nu_{n+1} f_{1}^{\nu_{1}} f_{2}^{\nu_{2}} \cdots f_{n+1}^{\nu_{n+1}} \equiv 0
$$

where in each term,

$$
\sum_{i=1}^{n+1} m_{i} \nu_{i} \leqq \prod_{i=1}^{n+1} m_{i}
$$

* Let every distributive functional on $S$ be linear, where $S$ is a topological vector space with the property $(Q)$. If $S$ is infinite dimensional, let $\left\{x_{n}\right\},(n=1,2, \cdots)$, be an infinite set of linearly independent elements. Since $\lim _{k \rightarrow \infty} k^{-1} x_{n}=\Theta$, we can choose $y_{n} \varepsilon S,(n=1,2, \cdots)$, linearly independent, with $y_{n} \rightarrow \Theta$. We set $f\left(y_{n}\right)=1, f(x)=0$ when $x$ is not a finite linear combination of the $y_{n}, f(a x+b y)=a f(x)+b f(y)$ for any $x \in S, y \varepsilon S$; then $f$ is a distributive functional on $S$, and hence is linear on $S$. Since $y_{n} \rightarrow \Theta, f\left(y_{n}\right) \rightarrow 0$ as $n \rightarrow \infty$; but this contradicts $f\left(y_{n}\right)=1$. Consequently $S$ is finite dimensional.

$\dagger$ Presented to the Society, February 25, 1939, under the title $A$ note on fundamental systems of symmetric functions.

$\ddagger$ O. Perron, Bemerkung zur Algebra, Sitzungsberichte der Bayerischen Akademie, mathematisch-naturwissenschaftliche Abteilung, 1924, pp. 87-101. 
The coefficients $C_{\nu_{1} \nu_{2}} \cdots v_{n+1}$ belong to the coefficient field of $f_{1}, f_{2}, \cdots, f_{n+1}$.

Consider any $n$ algebraically independent polynomials $\phi_{1}, \phi_{2}, \cdots, \phi_{n}$, of degrees $m_{1}, m_{2}, \cdots, m_{n}$, with coefficients in a field $K$ of characteristic zero. By Theorem 1 there exist relations

$$
\Phi_{i}\left(x_{i}, \phi_{1}, \phi_{2}, \cdots, \phi_{n}\right) \equiv 0, \quad i=1,2, \cdots, n,
$$

each of degree less than or equal to $\prod_{i=1}^{n} m_{i}$ in $x_{i}$. The algebraic independence assures the actual presence of $x_{i}$ in (1). It follows from (1) that the field $K\left(x_{1}, x_{2}, \cdots, x_{n}\right)$ of all rational functions of the $x_{1}, x_{2}, \cdots, x_{n}$ is a finite algebraic extension of the field $K\left(\phi_{1}, \phi_{2}, \cdots, \phi_{n}\right)$ generated by $\phi_{1}, \phi_{2}, \cdots, \phi_{n}$. Since $K$ is of characteristic zero, this extension contains a primitive element $\xi$, which, by Theorem 1, satisfies a relation of the type (1) of degree less than or equal to $\prod_{i=1}^{n} m_{i}$ in $\xi$. Hence we have the following lemma:

LEMmA 1. If $\phi_{1}, \phi_{2}, \cdots, \phi_{n}$ are $n$ algebraically independent polynomials of degrees $m_{1}, m_{2}, \cdots, m_{n}$, then the field $K\left(x_{1}, x_{2}, \cdots, x_{n}\right)$ is a finite algebraic extension of $K\left(\phi_{1}, \phi_{2}, \cdots, \phi_{n}\right)$ of degree less than or equal to $\prod_{i=1}^{n} m_{i}$.

The following result, which we state as a lemma, is well known :*

LEMMA 2. If $a_{1}, a_{2}, \cdots, a_{n}$ are the elementary symmetric functions of $x_{1}, x_{2}, \cdots, x_{n}$, then $K\left(x_{1}, x_{2}, \cdots, x_{n}\right)$ is a Galois extension of $K\left(a_{1}, a_{2}, \cdots, a_{n}\right)$ of degree $n$ !.

Suppose now that $\phi_{1}, \phi_{2}, \cdots, \phi_{n}$ are algebraically independent and symmetric. Since $a_{1}, a_{2}, \cdots, a_{n}$ form a fundamental system of symmetric functions, it is clear that $K\left(a_{1}, a_{2}, \cdots, a_{n}\right)$ contains $K\left(\phi_{1}, \phi_{2}, \cdots, \phi_{n}\right)$. Hence the degree of $K\left(x_{1}, x_{2}, \cdots, x_{n}\right)$ over $K\left(\phi_{1}, \phi_{2}, \cdots, \phi_{n}\right)$ must be a multiple of the degree of $K\left(x_{1}, x_{2}, \cdots, x_{n}\right)$ over $K\left(a_{1}, a_{2}, \cdots, a_{n}\right)$. If $\prod_{i=1}^{n} m_{i}<2 n$ !, it follows from Lemma 1 that the degree of $K\left(x_{1}, x_{2}, \cdots, x_{n}\right)$ over $K\left(\phi_{1}, \phi_{2}, \cdots, \phi_{n}\right)$ must be $n$ !. Hence

$$
K\left(\phi_{1}, \phi_{2}, \cdots, \phi_{n}\right)=K\left(a_{1}, a_{2}, \cdots, a_{n}\right),
$$

and we have the theorem:

Theorem 2. Any set of $n$ algebraically independent polynomials $\phi_{1}, \phi_{2}, \cdots, \phi_{n}$, symmetric in $x_{1}, x_{2}, \cdots, x_{n}$, over a field of characteristic zero forms a fundamental system if the product of their degrees is less than $2 n !$.

\footnotetext{
* Cf. van der Waerden, Moderne Algebra, vol. 1, p. 173.
} 
Theorem 2 is the best possible theorem of its kind; that is, the best general sufficiency condition for a fundamental system in terms of an upper bound for the product of the degrees without reference to the form of the polynomials $\phi_{1}, \phi_{2}, \cdots, \phi_{n}$. This may be verified by the example $\phi_{1}=a_{2}, \phi_{i}=S_{i},(i \geqq 2)$, where $a_{2}$ is the elementary symmetric function of degree 2 , and $S_{i}$ is the sum of the $i$ th powers of the variables. In this case, the product of the degrees is $2 n$ !. The independence of $\phi_{1}, \phi_{2}, \cdots, \phi_{n}$ is established by showing the nonvanishing of the functional determinant $D$. The expression for $D$ is

$$
D=n ! \cdot\left|\begin{array}{cccc}
a_{1}-x_{1} & a_{1}-x_{2} & \cdots & a_{1}-x_{n} \\
x_{1} & x_{2} & \cdots & x_{n} \\
2 & 2 & \cdots & x_{n}^{2} \\
x_{1} & x_{2} & \cdots & x^{n-1} \\
\cdot & \cdot & \cdots & \cdot \\
x_{1}^{n-1} & x_{2}^{n-1} & \cdots & x_{n}^{n}
\end{array}\right|
$$

where $a_{1}=x_{1}+x_{2}+\cdots+x_{n}$. After adding the second row to the first, and factoring $a_{1}$ from the first row, we have the Vandermonde determinant. Hence $D$ does not vanish identically. On the other hand, $a_{1}=\left(\phi_{2}+2 \phi_{1}\right)^{1 / 2}$ is an irrational expression for $a_{1}$ whose uniqueness is guaranteed by the independence. In other words, $a_{1}$ cannot be expressed rationally in terms of the set $\phi_{1}, \phi_{2}, \cdots, \phi_{n}$, and the latter set does not form a fundamental system.

YALE UNIVERSITY 